ARTíCULOS DE INVESTIGACIÓN

\title{
Estrategia pedagógica para la enseñanza de la Constitución Política en la construcción de competencias ciudadanas en Vista Hermosa, Meta, Colombia
}

\author{
Estratégia pedagógica para o ensino da Constituição Política na construção \\ da consciência cidadã em Vista Hermosa, Meta, Colômbia
}

\section{Pedagogical strategy for teaching about the Political Constitution in the construction of citizenship competencies in Vista Hermosa, Meta, Colombia}

\author{
Yudy Andrea Carrillo Cruz iD y Laura Mercedes Marín Ochoa iD \\ Universidad Cooperativa de Colombia
}

\begin{abstract}
RESUMEN El objetivo de este estudio fue crear una estrategia pedagógica para la enseñanza de la Constitución Política de Colombia, que aportara a la construcción de competencias ciudadanas en los niños del Municipio de Vista Hermosa, Meta, el cual, antes del proceso de paz de 2016, sufrió, de manera directa, la violencia de los grupos armados al margen de la ley. El método utilizado se implementó a través de la plataforma Cuadernia, que tiene licencia creative commons. Así, se elaboraron actividades lúdicas para el aprendizaje de los principios y de los derechos consagrados en la Constitución Política de 1991. Participaron 59 estudiantes de grado cuarto y quinto de primaria del Centro Educativo los Centauros, sede La Esperanza, entre nueve y doce años. En los resultados se demostró que, a través de la plataforma digital y las actividades diseñadas, los niños aprendieron conceptos básicos constitucionales y se entregó a los profesores de esta región una herramienta útil para la enseñanza de la Constitución Política. A modo de conclusión, se puede decir que la estrategia pedagógica utilizada se puede replicar en otras regiones de Colombia, principalmente en esta época de pandemia, ya que no requiere internet y contribuye al cumplimiento del artículo 41 de la Constitución.
\end{abstract}

PALABRAS CLAVE Constitución política, método de enseñanza, competencias ciudadanas, plataforma digital, práctica pedagógica.

RESUMO o objetivo deste estudo foi criar uma estratégia pedagógica para o ensino da 
Constituição Política da Colômbia, que contribuísse para a formação da consciência cidadã nas crianças do Município de Vista Hermosa, Meta, que antes do processo de paz, iniciado em 2016, sofreram diretamente a violência de grupos armados que atuavam ilegalmente. Método: por meio da plataforma Cuadernia, que possui licença creative commons, foram desenvolvidas atividades recreativas para o ensino dos princípios e direitos consagrados na Constituição Política de 1991. Participaram 59 alunos, entre 9 e 12 anos, do $4^{\circ}$ e $5^{\circ}$ anos do ensino primário do Centro Educacional Los Centauros, sede La Esperanza. Resultados: restou demonstrado que por meio da plataforma digital e das atividades projetadas, as crianças aprenderam conceitos constitucionais básicos e uma ferramenta útil para o ensino da Constituição Política foi entregue aos professores da região. Conclusão: A estratégia pedagógica utilizada pode ser replicada em outras regiões da Colômbia, principalmente neste momento de pandemia, uma vez que não requer internet e contribui para o cumprimento do artigo 41 da Constituição.

PALAVRAS-CHAVE Constituição política, método de ensino, consciência cidadã, plataforma digital, prática pedagógica.

ABSTRACT The objective of this study was to create a pedagogical strategy for the teaching of the Political Constitution of Colombia, which would contribute to the construction of citizenship competencies in the children of the Municipality of Vista Hermosa, Meta, which, before the peace process of the year 2016, suffered directly the violence of armed groups outside the law. Method: through the Cuadernia platform, which has a creative commons license, recreational activities were developed to learn the principles and rights enshrined in the 1991 Political Constitution. 59 students from the fourth and fifth grade of elementary school from the Los Centauros Educational Center participated. La Esperanza headquarters, between 9 and 12 years old. Results: it was demonstrated that through the digital platform and the activities designed, the children learned basic constitutional concepts and an useful tool for teaching the Political Constitution was given to the teachers of this region. Conclusion: The pedagogical strategy used can be replicated in other regions of Colombia, mainly in this time of pandemic, since it does not require internet and contributes to compliance with article 41 of the Constitution.

KEYWORDS Political constitution, teaching method, civic competences, digital platform, pedagogical practice.

\section{Introducción}

Vista Hermosa es el nombre que recibe un municipio localizado en la parte sur del Departamento del Meta en la República de Colombia. Se encuentra aproximadamente a seis horas por carretera de Bogotá Distrito Capital, a una distancia de 264 kilómetros. ${ }^{1}$ Es un territorio principalmente rural, con exuberante vegetación y diversidad

1. «Municipio», Alcaldía De Vista Hermosa, disponible en https://bit.ly/3HeGuZI. 
de fuentes hídricas. Junto con otros municipios conforma el Parque Natural Sierra de la Macarena y, durante el conflicto interno del país, fue uno de los lugares más afectados por los grupos al margen de la ley, entre ellos el antiguo grupo Fuerza Armada Revolucionaria de Colombia (FARC-EP), quienes plantaron allí cultivos ilícitos y tomaron el control por la vía armada (Tobón y Restrepo, 2011: 31).

Las consecuencias de la violencia se han visto reflejadas en su sistema educativo, que solo tiene planteles de básica primaria y secundaria; en la falta acceso a la tecnología, ya que no hay servicio a internet estable y las condiciones tanto económicas como sociales son difíciles para estudiantes y profesores. Para que los estudiantes accedan a la educación superior, el lugar más cercano es la ciudad de Villavicencio a 157 kilómetros. $^{2}$

Al firmarse, en noviembre de 2016, el Acuerdo de Paz entre el gobierno nacional, en cabeza del Nobel de la Paz de aquel año, Juan Manuel Santos, y la antigua guerrilla FARC-EP, ${ }^{3}$ Colombia entró en un proceso de posconflicto que dejó al descubierto las necesidades y los problemas que enfrentaban los territorios más vulnerables por la situación de violencia que allí vivían (Capera y Ñañes, 2017). Asimismo, la desmovilización del grupo armado permitió que la comunidad académica cercana a Vista Hermosa pudiera ingresar para apoyar a la población en los procesos de reconciliación.

Los resultados de este artículo derivan de un proyecto creado en la Facultad de Derecho de la Universidad Cooperativa de Colombia, campus Villavicencio, que decidió aportar a la enseñanza de la Constitución Política de 1991 a los niños de grado cuarto y quinto del Centro Educativo los Centauros, sede la Esperanza, con el fin de incentivar la educación para la paz y las competencias ciudadanas. Para eso, se diseñó una metodología que acercara a los infantes a la norma constitucional.

El grupo de investigación partió de teorías, como la expuesta por la investigadora Sandra Hincapié, quien comparó la democracia local en contextos de violencia en Colombia y México, y llegó a la conclusión de que, en las zonas en las que hay contextos de violencia, se ve aminorada la democracia local, y si no se realizan acciones colectivas que permitan procesos de cambio, las personas pueden ser fácilmente víctimas de una acción violenta en su contra (Hincapié, 2017: 84). En ese entendido, el programa de derecho consideró importante contribuir a la construcción de competencias ciudadanas que lograran transformar la historia de las personas que viven en regiones que sufrieron la violencia en Colombia, $y$ un componente importante en esta construcción es la enseñanza de la Constitución Política a los niños, de quienes se espera actúen como multiplicadores de estos aprendizajes para la paz.

\footnotetext{
2. «Municipio».

3. Natalio Cosoy, «Colombia: Gobierno y las FARC firman un nuevo acuerdo de paz». BBC Mundo, 13 de noviembre de 2016, disponible https://bbc.in/3mFUkws.
} 


\section{La importancia de la educación para la paz en el posconflicto de Colombia}

La intensidad de la violencia en Colombia no fue igual en todo el territorio, como tampoco lo ha sido en todas las épocas de su historia (Uribe, 2019). Las consecuencias del conflicto armado fueron diferentes para la población ubicada en zonas rurales y urbanas. En el área rural, como Vista Hermosa, la presencia de grupos al margen de la ley era intimidante, imponían decisiones a las comunidades, que, en sentido práctico, anulaban la existencia del Estado y de los gobernantes legítimamente constituidos (Trigos, Fonseca y Reinoso, 2020). La participación de los ciudadanos en las elecciones en calidad de candidatos o electores era menoscaba por la presión que ejercían los grupos armados, desconociéndose el principio democrático y los derechos políticos (Ávila y Velasco, 2012).

Después de la firma del Acuerdo de Paz de 2016, surgió un período de transición para lograr su implementación, el cual se denominó posconflicto (Acevedo y Rojas, 2016: 43). Actualmente, aún se están implementando los acuerdos convenidos entre los dos grupos negociantes. Este período histórico ha sido muy valioso en Colombia para acercarse a las comunidades rurales, que no solo fueron marginadas debido al conflicto armado, sino también por las condiciones geográficas. Estas razones han impedido un desarrollo tecnológico que permita a la educación beneficiarse de herramientas digitales en el proceso de aprendizaje. De ahí la importancia de las plataformas digitales que no exigen el uso de internet, como el caso de la utilizada en este proyecto, y que aportan a la formación de los estudiantes del sector rural.

Académicos como María Liliana Díaz y Nelson Darío Rojas, en el artículo «Educación para la ciudadanía en el posacuerdo», rastrearon investigaciones sobre la forma en que se ha desarrollado la formación ciudadana en períodos de posconflicto en algunos países que han pasado por estas etapas en su historia política y social, y concluyeron que «mientras la educación conserve parámetros violentos, autoritarios, con modelos pedagógicos basados en la memoria, no es posible aclimatar una cultura de paz» (Díaz y Rojas, 2018: 1).

El posconflicto exige la implementación de una educación para la paz, que forme ciudadanos capaces de crear o reconstruir relaciones sociales armoniosas basadas en el consenso y no en la violencia, y con el reconocimiento de medios pacíficos para resolver los conflictos sociales (Pérez, 2014: 303).

Antes del Acuerdo, ya el Congreso de la República de Colombia había emitido la Ley 1.732 de 2015, que fue reglamentada por el Decreto 1.038 ese mismo año, en el que se implementa la Cátedra de la Paz. Textualmente, en su artículo 2, el Decreto prescribe como tema de esta cátedra lo siguiente: «Educación para la paz: se entiende como la apropiación de conocimientos y competencias ciudadanas para la convivencia pacífica, la participación democrática, la construcción de equidad, el respeto por la pluralidad, los derechos humanos y el derecho internacional humanitario». 
La educación para la paz quedó consagrada en el segundo punto del Acuerdo de Paz entre las FARC-EP y el Estado colombiano, en el marco del reconocimiento de la oposición política y la divergencia de opiniones (Cediel, Olave y Cisneros, 2019). Textualmente, el acuerdo señala la «Creación de una cátedra de cultura política para la reconciliación y la paz (Gobierno de Colombia y FARC-EP, 2016: 47).

Una de las grandes dificultades para lograr la paz en un Estado como Colombia es la negativa de reconocer al otro como un adversario político legítimo (Cediel y otros, 2019: 24).

Una cátedra de paz es urgente en Colombia, no en vano así lo han reconocido tanto el gobierno nacional como los representantes de la que fuera la guerrilla más antigua del mundo. Es necesario que los niños colombianos comprendan la importancia del respeto por la diferencia, que aprendan de la existencia de las instituciones democráticas, de los mecanismos de participación ciudadana, que interioricen sus derechos constitucionales y los hagan exigibles a través de los mecanismos jurídicos existentes para su defensa, y que adviertan que los demás también tienen derechos.

\section{La Constitución Política como fundamento en la construcción de competencias ciudadanas}

La norma fundamental en Colombia es la Constitución Política de 1991, producto de un consenso deliberativo en la cual se consagra una parte dogmática y una parte orgánica (Leiva, Jiménez y Meneses, 2019). El articulado de la parte dogmática comprende «los principios filosóficos que orientan la organización estatal y definen las relaciones básicas entre los ciudadanos y las autoridades» (Corte Constitucional, 2002).

La Constitución Política (2021) prescribe, en su artículo 41, que:

En todas las instituciones de educación, oficiales o privadas, serán obligatorios el estudio de la Constitución y la instrucción cívica. Asimismo, se fomentarán prácticas democráticas para el aprendizaje de los principios y valores de la participación ciudadana. El Estado divulgará la Constitución.

El proceso de paz trajo consigo una movilización de la academia en pro de incentivar la cultura de la no violencia en el país. Es imposible lograr que en la sociedad no existan ideologías diversas e incluso antagónicas, sin embargo, la manera de resolver los conflictos dice mucho de la formación ciudadana de la población de un Estado.

Se realizaron diversidad de foros, encuentros, simposios y congresos académicos a lo largo y ancho del territorio nacional promoviendo la reconciliación y debatiendo cada tema acordado en La Habana, lugar donde se llevaron a cabo los diálogos (Rodríguez Pico, 2015). La comunidad académica colombiana se sintió con la responsabilidad de aportar a la construcción de la paz. Producto de estas discusiones, se concluyó que la falta de conocimiento de los derechos constitucionales y el descono- 
cimiento sobre el funcionamiento del Estado no permite que la población realmente viva verdaderos procesos democráticos; entendiéndose por democracia no solo el hecho de participar en las urnas mediante el voto, sino en el sentido que lo entiende Habermas, donde la población es libre de deliberar y llegar a consensos sin arriesgar su integridad.

Un aporte significativo que puede hacer la academia en la construcción de competencias ciudadanas en aquellos lugares que vivieron de manera directa la violencia en Colombia es ayudar a su población a conocer la Constitución Política del Estado. Por esta razón, se formuló la pregunta de investigación: ¿qué metodología se puede diseñar para la enseñanza de la Constitución Política de Colombia en los grados cuarto y quinto de básica primaria, de la Institución Educativa los Centauros sede la Esperanza del Municipio de Vista Hermosa, Meta, ¿con el fin de que los estudiantes comprendan los principios del Estado y sus derechos fundamentales?

Este artículo divulga la técnica pedagógica diseñada y empleada para enseñar la Constitución Política colombiana de una manera agradable y apropiada a los estudiantes de Vista Hermosa que hicieron parte de la investigación.

Normalmente, la Constitución Política se enseña a través de exposiciones y lecturas guiadas. A nivel universitario se usan casos jurisprudenciales, pero, en este caso, se debía tener en cuenta que la población beneficiada tenía entre nueve y doce años, por lo que estos métodos podrían no ser llamativos ni comprensibles para ellos y se pretendía que los estudiantes aprendieran de manera lúdica, utilizando una herramienta que generara diversión y, al mismo tiempo, que ayudara a la comprensión de la temática.

El reto era crear una herramienta pedagógica para enseñar la Constitución Política principalmente en la infancia. La hipótesis formulada concluía que aprender la Constitución podría ayudar a los niños a formar competencias ciudadanas que permitieran su inclusión en la sociedad de manera proactiva y no de forma violenta.

El método pedagógico empleado logró que los niños participantes en la investigación comprendieran sus derechos y el respeto de los derechos de los otros, así como conceptos básicos de la organización del Estado establecidos en los principios constitucionales.

La investigadora Elizabeth Leonor Zambrano (2018: 70) piensa que las prácticas pedagógicas son aquellas «estrategias, instrumentos y acciones que el docente realiza en el aula para guiar el proceso de enseñanza-aprendizaje, con la pretensión de desarrollar en el educando diversas competencias».

José Antonio Marina y Bernabeu Merlo (2014) sostienen que:

Noam Chomsky popularizó la noción de competencia al usarla para explicar el aprendizaje infantil del lenguaje. Diferenciaba entre competencia y actuación (performance) lingüística: mientras que la competencia indica una capacidad potencial, 
determinada por el grado de maduración biológica, la actuación consistía en la realización efectiva de ese potencial.

Formar en competencias ciudadanas trasciende a solo adquirir conocimientos. De acuerdo con el Ministerio de Educación Nacional de Colombia, en la «Guía 6: Estándares Básicos de Competencias Ciudadanas» (2004: 168), estas competencias requieren del concurso, además, de competencias comunicativas, cognitivas, emocionales e integradoras. Las competencias, «articuladas entre sí, hacen que el ciudadano democrático esté dispuesto a actuar de manera constructiva y justa en la sociedad (Ministerio de Educación Nacional de Colombia, 2004: 8).

La investigación no estaba direccionada a formar de manera integral a los estudiantes en todas sus competencias ciudadanas, sino a aportar una herramienta pedagógica para incentivar el área cognitiva, específicamente frente al estudio de la Constitución Política. Chaux (citado por Luquez y otros, 2012: 485) menciona que los conocimientos en competencias ciudadanas se refieren a la comprensión de información, procedimientos, estrategias y contenidos, y al conocimiento de los derechos fundamentales. Tener habilidades cognitivas logra que se tenga la capacidad de realizar procesos mentales e imaginarse diferentes maneras de resolver un conflicto o una problemática social.

Por otra parte, es importante considerar que Chaux (citado por Betancourt, Bernate y Fonseca, 2020: 846) aclara que:

La competencia ciudadana hace parte en el desarrollo democrático de una nación, y el compromiso de formar ciudadanos respetuosos de las diferencias, que tengan una orientación a la sana convivencia, solidarios y con múltiples herramientas en la resolución de conflictos, entre otras muchas habilidades.

Chaux (2009), en el artículo «Citizenship competencies in the midst of a violent political conflict: The Colombian educational response», considera el efecto que un ambiente repleto de conflictos políticos violentos tiene sobre los niños. Estas investigaciones soportan estrategias que quieren aminorar los efectos de la violencia en los niños de las zonas rurales de Colombia para que sean adultos con las competencias ciudadanas necesarias para convivir en sociedad.

Si bien las competencias ciudadanas no solo se forman en la escuela, sino que comprenden otros escenarios como la familia, el pueblo, la sociedad. Así, como lo reconoce Chaux y otros (2004: 10), la escuela se convierte en un espacio privilegiado para la formación ciudadana, de ahí que surjan propuestas para trabajar en el ámbito escolar.

Otros estudios han analizado estrategias pedagógicas para la formación de competencias ciudadanas en los espacios académicos de primaria, secundaria y a nivel universitario. Todo esto indica que la construcción de las competencias ciudadanas inicia en la infancia, pero continúa a lo largo de la vida hasta la adultez. 
Las investigadoras Laura Puerta y Yaneth Urrego (2016: 129) llevaron a cabo un estudio en las ciudades de Barranquilla y Bogotá, en el que diseñaron, aplicaron y evaluaron el programa llamado Funciones Ejecutivas de Intervención para la Promoción de Competencias Ciudadanas (FEJINCOC) para estudiantes de básica primaria. Este programa fue desarrollado principalmente para estimular las funciones de flexibilidad cognitiva, fluidez verbal, inhibición, iniciativa, creatividad, memoria de trabajo, planificación y toma de decisiones.

A nivel superior igualmente se han desarrollado investigaciones entre los estudiantes de diferentes universidades de Colombia. Alicia Álvarez, Omar Sandoval y Mildred Puello (2018: 7) evidenciaron que era necesario implementar estrategias que posibilitaran el desarrollo de competencias ciudadanas en estudiantes de derecho de la Universidad de la Costa en Colombia, de manera que se mostrara coherente su comportamiento, porque no se veían reflejadas las competencias ciudadanas en su vida cotidiana. En esta misma institución universitaria se llevó a cabo un proyecto para desarrollar las competencias ciudadanas de los jóvenes universitarios en los cursos de Constitución Política, Ética y Taller de Pensamiento, toda vez que son conscientes de que, si se quiere una sociedad en paz, es necesario que la educación superior también involucre la formación de las competencias ciudadanas (Maussa, 2015: 573).

En una investigación llevada a cabo en la Universidad del Magdalena, (Zambrano, 2018: 80) diseñaron una serie de prácticas pedagógicas constructivas, consistentes en estudio de casos; y otras lúdicas, como los juegos, para la adquisición de competencias ciudadanas. Se concluyó que obtuvieron mejores resultados los estudiantes que aprendieron, a través de las prácticas, estrategias lúdicas, ya que, en las prácticas netamente expositivas, el estudiante no puede manifestar sus emociones, impidiendo que se puedan desarrollar las competencias. Conocer estas experiencias reforzaron la hipótesis de que los niños podían comprender mejor la Constitución si se les explicaba a través de juegos y actividades lúdicas.

El aprendizaje que obtuvieron los niños que participaron en esta estrategia pedagógica los beneficiará en su edad adulta, incluso en su área laboral. Así lo han demostrado estudios como el realizado por los chilenos Olga Carrillo Mardones y Patricio Lagos y el español Pedro Jurado de los Santos (2018), quienes afirman que es importante la formación en competencias ciudadanas «en la formación profesional, ya que se presentan como facilitadoras de la inserción al mercado laboral».

Martha Ochman y Jesús Cantú (2013) llevaron a cabo un proyecto para evaluar el nivel del desarrollo de las competencias ciudadanas en los estudiantes de educación media superior y superior en México, en el cual diseñaron una herramienta de evaluación de manera sistemática y objetiva. Si bien la investigación aportaba al estado del arte de este proyecto, la intención en Vista Hermosa no era evaluar el nivel de desarrollo de las competencias ciudadanas de los niños y niñas participantes en la 
investigación, sino crear y aplicar una técnica didáctica con la cual se amplíen los conocimientos que tenían sobre la Constitución Política.

\section{Hipótesis y metodología}

La investigación utilizó el método cualitativo con el fin de entender el contexto cultural, histórico y social en el cual estaba inmersa la población participante. La hipótesis que se logró demostrar fue que sí es posible diseñar una herramienta pedagógica para el estudio de la Constitución Política de 1991 que apoye la construcción de competencias ciudadanas cognitivas en los niños de los grados cuarto y quinto del Centro Educativo Los Centauros, sede La Esperanza, en el municipio de Vista Hermosa, Meta. Así, la metodología sería viable para que se aplique en otras instituciones educativas colombianas.

La técnica pedagógica diseñada para la enseñanza de la Constitución fue utilizada con los estudiantes del Centro Educativo Los Centauros, ubicado en la zona urbana de Vista Hermosa, Meta, cuya muestra se delimitó a 59 estudiantes de la sede de educación primaria llamada La Esperanza, 31 de grado cuarto y 28 de grado quinto, a quienes sus padres autorizaron expresamente a través de un documento legal y, asimismo, firmaron el asentimiento que se requiere, por razones éticas, para este tipo de investigaciones.

\section{Instrumento}

Como quiera que la herramienta pedagógica producto de la investigación pueda ser utilizada en otras instituciones educativas, en este artículo se va a detallar la metodología empleada para su diseño, aplicación y los resultados obtenidos.

Es importante señalar que, si bien el estudio de la Constitución Política no es exclusivo de los abogados, teniendo en cuenta que el artículo 41 constitucional ordena la enseñanza de esta norma a toda la población colombiana, sí es verdad que quienes más profundizan en su historia, estructura, importancia, interpretación y aplicación son los estudiantes de derecho. Ahora bien, estudiosos como Jorge Castellanos (2020) sostienen que el buen uso de las tecnologías de la información en la enseñanza del derecho puede ser muy útil para formar juristas con una visión integral de la realidad jurídica, lo que significa que sí puede ser posible utilizar las tecnologías de la información en la enseñanza de la carta magna del Estado.

La investigación empleó, como herramienta tecnológica para la enseñanza de la Constitución, el programa Cuadernia, creado por la Universidad de Castilla-La Mancha en España, cuya licencia creative commons permite usarlo de manera gratuita. Uno de los limitantes de los estudiantes y profesores de derecho es que sus competencias no les permiten crear un programa digital original, pero legalmente sí pueden utilizar Cuadernia. 
La plataforma puede ser descargada desde la página web de la Universidad de Castilla-La Mancha y ser usada sin necesidad de internet, cualidad que determinó su elección por cuanto el proyecto estaba siendo desarrollado en un lugar donde no hay buena conectividad a internet. Adicionalmente, una vez se organizadas las actividades, se puede grabar la aplicación en todos los ordenadores que se vayan a destinar para la enseñanza, siendo muy valioso para que el programa sea funcional en otras instituciones educativas en Colombia.

El proyecto fue denominado Plataforma Interactiva de la Constitución Política (PICOP), la cual se construyó teniendo en cuenta que el programa Cuadernia admite el uso de imágenes propias, por lo que, en este caso, se emplearon los dibujos que los niños crearon después de ser impartidas las enseñanzas por parte de los estudiantes y profesores de derecho sobre los principios constitucionales y los derechos fundamentales. Igualmente, se crearon actividades lúdicas como rompecabezas, crucigramas, sopas de letras y cuestionarios, entre otras, para reforzar los conocimientos aprehendidos de manera lúdica y divertida.

La figura 1 muestra el diseño que tiene la plataforma, en la cual se observa un dibujo realizado por uno de los estudiantes participantes y una de las actividades implementadas para entender los principios constitucionales.

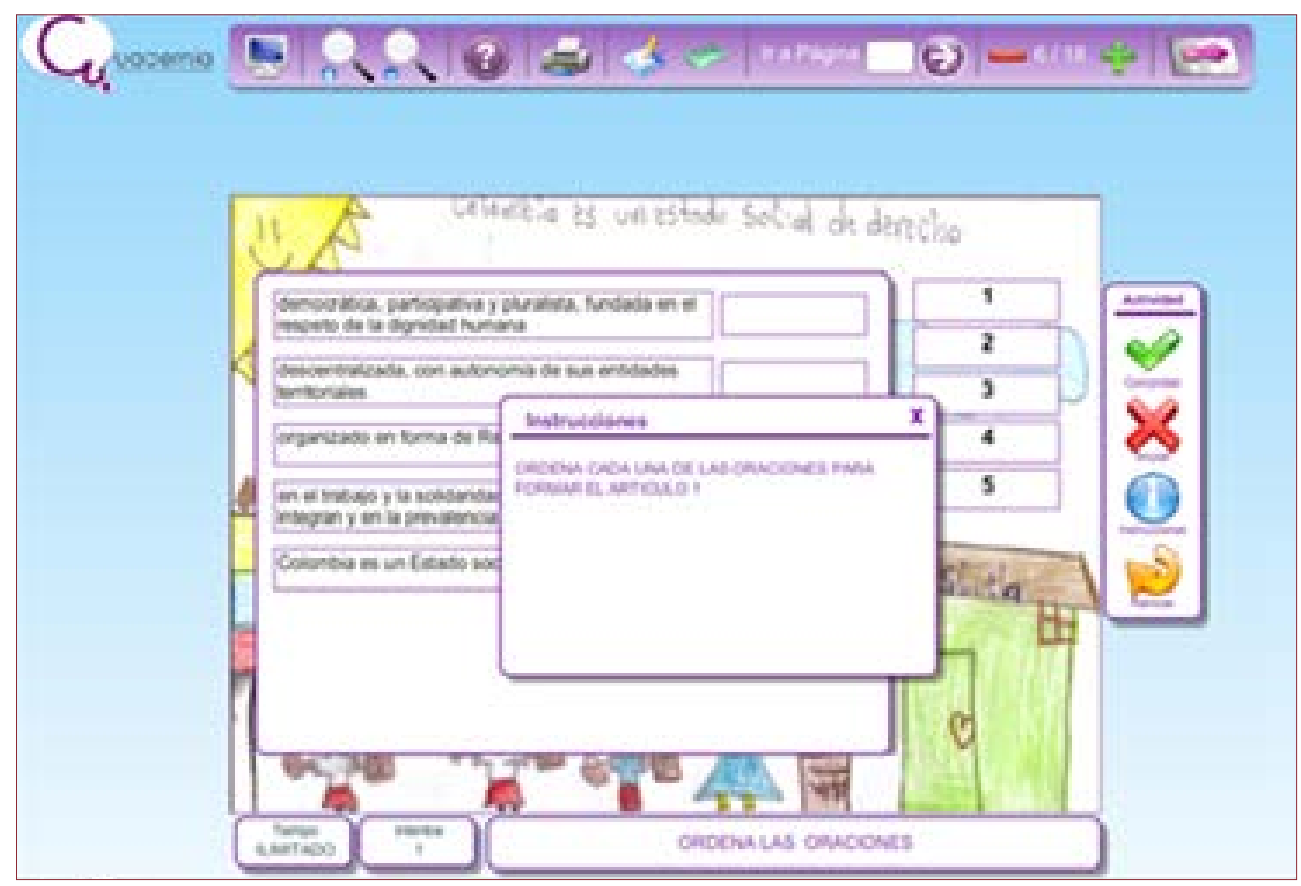

Figura 1. Imagen de la plataforma digital para enseñar la Constitución Política de Colombia. Fuente: Fotografía de los semilleristas Leonar Lucero Sánchez y Julián Morales Acosta 


\section{Aplicación del instrumento y análisis de datos}

Antes de aplicar la metodología PICOP se requiere saber el nivel de conocimiento que tienen sobre la Constitución Política y otros conceptos del Estado, por lo cual se debe aplicar una encuesta inicial. Los 59 estudiantes participantes respondieron las preguntas para saber si conocían la Constitución Política de 1991, si sabían qué era un derecho fundamental, qué era el Estado y su estructura, si entendían el significado de la palabra democracia y si les gustaría aprender sobre sus derechos fundamentales y la manera en cómo funcionaba el Estado colombiano.

El 100\% de los estudiantes señalaron que no conocían la Constitución Política, no tenían claro qué significaba la palabra Estado, ni su estructura, dijeron no saber sobre la democracia y no tenían claros los derechos fundamentales. No obstante, todos los niños contestaron que sí les gustaría conocer sus derechos, saber sobre el Estado y estuvieron muy interesados en el proceso de aprendizaje.

En las primeras visitas realizadas por parte de los profesores y estudiantes investigadores a la institución educativa La Esperanza, se explicaron los temas que, se pretendían, fueran identificados por los estudiantes en la plataforma Cuadernia. Es importante que se transmita el conocimiento de manera sencilla. En este caso, se utilizaron carteleras, títeres, dinámicas en clase y se les solicitó a los estudiantes que, a través de dibujos, expresaran lo que habían entendido con respecto a los artículos constitucionales relacionados con los principios del Estado que están consagrados en los artículos 1 al 10 de la Constitución y los derechos fundamentales que están escritos desde el artículo 11 hasta el artículo 41 constitucional.

La técnica pedagógica de dibujar no fue seleccionada al azar. Víctor Gómez Lombart y Valentín Gavidia Catalán (2015: 443), en su artículo «Describir y dibujar en ciencias: La importancia del dibujo en las representaciones mentales del alumnado», se basan en «la teoría de los modelos mentales de Johnson-Laird (1983) que considera que la mente humana opera con tres tipos de representaciones mentales, distinguiendo proposiciones, modelos mentales e imágenes». De acuerdo con la concepción de imágenes, los estudiantes, al dibujar, están plasmando una visión de las representaciones mentales que tienen. Así las cosas, si el dibujo efectivamente logra dar un mensaje adecuado sobre el concepto que quiere explicar, eso significa que el estudiante sí comprende el tema y es capaz de representarlo, como se observó en muchos de los dibujos realizados.

$\mathrm{Al}$ estar listas las actividades lúdicas en la plataforma PICOP, se les explicó su uso a las profesoras y se grabó en los computadores con los cuales contaba la institución educativa. Debido a su escaso número, los estudiantes trabajaron en grupos de tres personas por computador y la experiencia de tener que jugar por turnos no tuvo inconveniente. Ellos estaban dispuestos a compartir los computadores con sus compañeros, situación que reflejaba que sí tenían competencias ciudadanas básicas en el desarrollo de su aprendizaje. 
El proceso debe terminar con una encuesta final, en la que se evidencia el nivel de conocimiento que adquirieron los estudiantes luego de haber utilizado la herramienta pedagógica. Los resultados obtenidos en esta fase del proyecto fueron satisfactorios, teniendo en cuenta que las preguntas diseñadas no pretendían que los estudiantes repitieran de memoria conceptos, sino que demostraran sus conocimientos de manera general.

\section{Resultados}

Los resultados que aquí se plasman dan cuenta de la manera en la que la metodología utilizada sí fue eficaz para comprender conceptos constitucionales, que, a pesar de ser abstractos, fue posible verlos reflejados en los dibujos creados por los niños, por lo que se entiende que sí alcanzaron a elaborar una representación mental de los artículos constitucionales, y la plasmaron desde su realidad en los dibujos, que incluso, tenían frases muy elocuentes de lo que el niño había comprendido. Por ejemplo, al explicar el artículo 12 constitucional, que habla sobre el derecho a la integridad física, el niño Ángel David, de once años, pintó al lado izquierdo de la hoja a un niño y a una niña golpeados, con sus ojos morados y manchas rojas, evocando la sangre; dividió la hoja con una línea y al lado derecho dibujó los mismos niños, pero sin golpes y felices. En la parte baja de la hoja escribió «todos los niños tienen derecho a ser felices». Este tipo de dibujos dejaban entrever que los estudiantes sí lograban comprender sus derechos fundamentales.

La niña Danna Yulieth, de nueve años, al dibujar lo que entendía por el principio de estado social de derecho y el principio democrático, dibujó una niña en la escuela en la parte izquierda de la hoja, y en la parte derecha dibujó la misma niña frente a una urna que decía «vote». Si bien esta no es la única expresión concreta con las que se materializan estos principios, es importante para la formación de competencias ciudadanas que la niña se visualice eligiendo mediante el acto de votar y siendo parte del proceso democrático del Estado. Este ejemplo de una mujer que ejerce sus derechos políticos con naturalidad es el fruto de todo un proceso histórico que se puede analizar desde el punto de vista del constitucionalismo crítico, como lo examina Nilda Garay Montañez (2014) al mencionar que existe un constitucionalismo feminista que ha enarbolado el discurso de una igualdad real entre hombres y mujeres, y es significativo que las niñas se apropien de sus derechos no desde las teorías, sino desde la vida real.

Yessica Tatiana, de doce años, quien cursaba cuarto grado, al dibujar sobre los derechos de los niños, prescrito en el artículo 44 de la Constitución, plasmó dos niños y dos niñas felices en la escuela, y escribió frases como «viva el respeto para los niños» $y$ «las mujeres deben recibir respeto y todos los colombianos deben haber paz 
y respeto». En otro lado de la hoja, se observa «derecho a la educación». Son frases que efectivamente corresponden a la interpretación del artículo.

Existe, entre las teorías de la democracia, una en particular que está siendo muy estudiada en Colombia, principalmente como sustento teórico de la implementación del Acuerdo de Paz de 2016, y es el de la democracia agonista, cuya exponente principal es Chantal Mouffe, la cual no niega el conflicto político, sino que, por el contrario, como lo interpreta Pauline Colonna d'Istria (2016: 23), «la idea de un pluralismo sin antagonismos es para ella una quimera». Así las cosas, se entiende que en una democracia no va a existir un verdadero consenso, sino que hay un conflicto político que debe reconocerse y, por tanto, asimismo, se deben reconocer los actores del conflicto, quienes tendrán derecho a oponerse y existir dentro del juego político democrático. No es desconocido que la República colombiana tiene una democracia débil debido a la incapacidad que ha existido de resolver los conflictos por vías pacíficas, ya que históricamente está demostrado que tanto los grupos al margen de la ley como las instituciones estatales han utilizado la violencia para eliminar a sus oponentes o contradictores políticos, como lo han reflejado las sentencias en el sistema interamericano de derechos humanos, razón por la cual, en el artículo 12 constitucional, se prohíbe la tortura y la desaparición forzada.

Una vez explicado el concepto de democracia desde este enfoque teórico, Danny, al explicar el artículo 12 sobre la prohibición de la tortura, dibujó a dos niños: uno le muestra un cuchillo al otro. El niño amenazado tiene sus manos arriba en señal de indefensión y dice, a través de un globo: «tú no tienes derecho a torturarme». El niño violento tiene su mano estirada con el arma y responde, a través de otro globo: «es verdad». Todo esto, se puede ver en la figura 2. Teniendo en cuenta la realidad en la cual estaban creciendo los niños participantes del proyecto, quienes estaban constantemente observando actos violentos en su cotidianidad debido a la presencia de los grupos armados, el dibujo indicaba que el niño había comprendido que nadie tenía derecho a torturar a nadie, siendo este un gran avance en la construcción de sus competencias ciudadanas.

Todos los niños elaboraron dibujos. En la parte inferior de la hoja debían escribir su nombre y sus datos, como la edad y su curso. Algunos de ellos firmaron con nombres y apellidos, otros solamente con el nombre y otros omitieron su edad. Se les motivó a pintar bien porque estaban en un concurso. Así, los mejores dibujos serían seleccionados para ser la imagen de la plataforma PICOP. En la premiación se entregaron regalos y se seleccionaron los mejores dibujos para recrear las actividades lúdicas en la plataforma. Ver sus dibujos en el computador ayudaba a que los niños sintieran un mayor interés por hacer las actividades. 


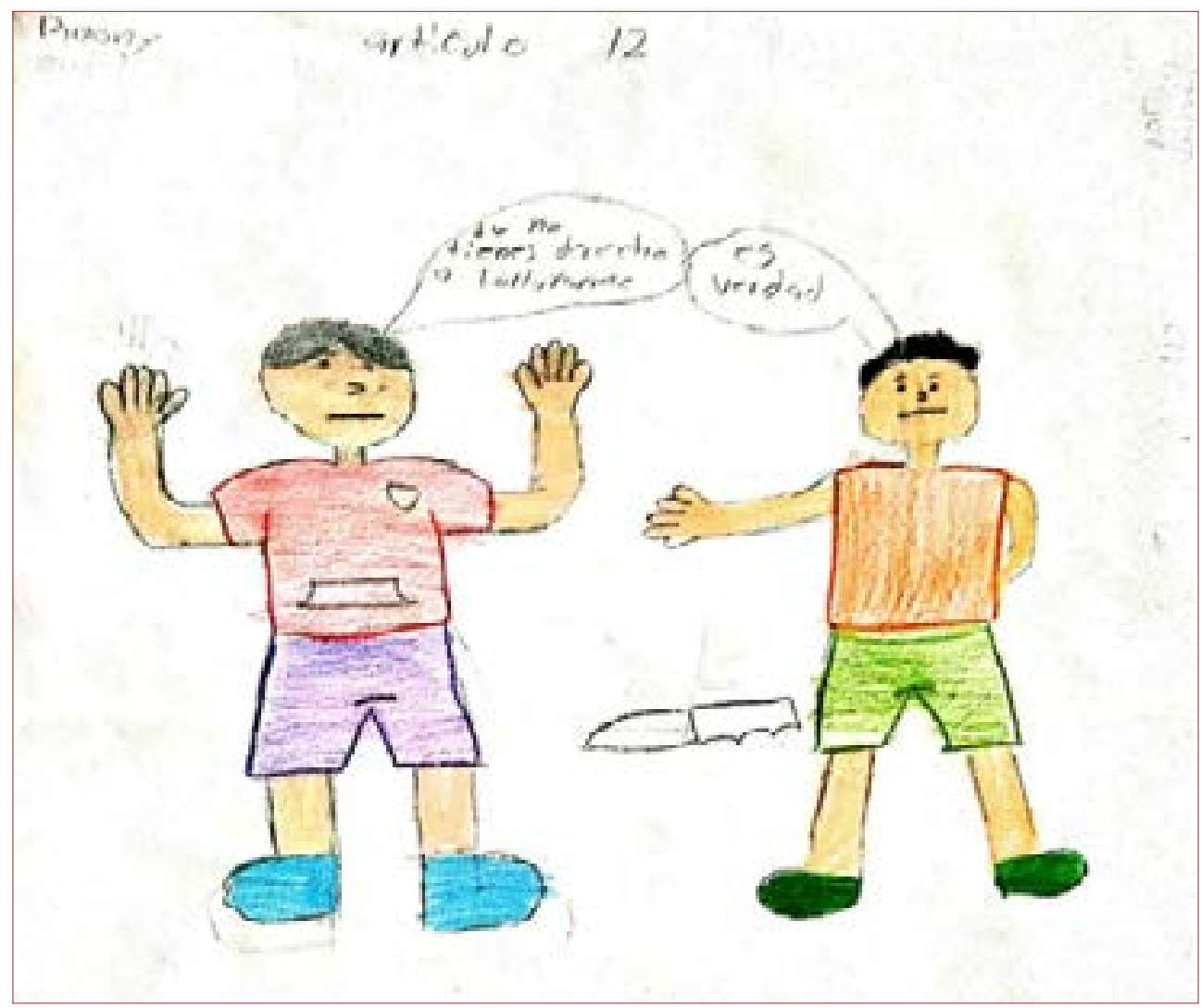

Figura 2. Dibujo realizado por Danny a propósito del artículo 12 sobre la prohibición de la tortura. Fuente: Fotografía semilleristas Leonar Lucero Sánchez y Julian Morales Acosta.

\section{Discusión y conclusiones}

Del análisis de los resultados se concluye que sí fue posible diseñar una metodología para enseñar la Constitución Política en los grados cuarto y quinto de básica primaria de la Institución Educativa los Centauros, sede la Esperanza, del Municipio de Vista Hermosa, Meta, con el fin de que los estudiantes comprendieran los principios del Estado y sus derechos fundamentales en Vista Hermosa, como lo demuestra la encuesta final en la que los niños, al contestar, señalaron la familiaridad con los conceptos aprendidos.

La metodología empleada es un aporte para la construcción de las competencias ciudadanas cognitivas en Colombia. Se encuentra disponible y se puede reproducir en otras instituciones educativas que quieran implementar el uso de tecnologías de la información en los procesos de enseñanza-aprendizaje de la norma fundamental del Estado. 
Las preguntas formuladas en el cuestionario final fueron cerradas para facilitar su comprensión y tabulación. Los estudiantes debían contestar con la expresión «sí» o «no». Se preguntó: ¿usted conoce la Constitución Política? ¿Usted conoce qué es un derecho fundamental? ¿Usted sabe qué es el Estado? ¿Usted sabe cuáles son los derechos de los niños? ¿Usted tiene derecho a la educación, al deporte, a la vida, a la salud, a una familia y al amor? ¿Usted tiene deberes con su familia y con el Estado?

El 100\% de los niños manifestó que conocían la Constitución Política del Estado colombiano. Por primera vez, la habían escuchado cercana, la habían leído en voz alta y habían jugado descubriendo la información de sus artículos. Tenían claridad sobre lo que era un derecho fundamental, sentían más familiaridad con el concepto Estado. El 100\% dijo conocerlo y se hicieron aclaraciones sobre la influencia que ejercían los grupos al margen de la ley en su territorio y cómo la paz aportaría a la tranquilidad y a tener vidas más felices. De igual forma, el 100\% de los estudiantes dijo saber los derechos fundamentales de los niños y no dudaron en afirmar que ellos tenían derecho a la educación, al deporte, a la vida, a la salud, una familia y al amor, entre otros.

Frente a la última pregunta, el $99 \%$ de los niños adujeron conocer los deberes que tienen con su familia y el Estado. No obstante, el 1\% tiene relación con un niño que mencionó no conocerlos.

Como lo señaló Castellanos (2020), la pandemia por el covid-19 demostró la importancia del uso de las tecnologías de la información en la enseñanza del derecho, lo que convierte a la herramienta digital PICOP en una metodología eficaz para aplicarse en todo el territorio nacional. No obstante, es importante mencionar que está pensada para utilizarse en las regiones que fueron epicentros de la violencia armada y en aquellas entidades territoriales que no cuentan con buen internet o conexión es inexistente.

Prueba de su eficacia es que esta misma metodología se llevó a otras instituciones educativas del sector rural del Departamento del Meta y se entregó el programa a los profesores para que los estudiantes pudieran aprender jugando, dando buenos resultados en dichas instituciones en el proceso de aprendizaje de la Constitución.

La experiencia con la plataforma digital PICOP, diseñada con Cuadernia, fue positiva para la enseñanza de la Constitución Política, y demuestra que el derecho no es ajeno al uso de las tecnologías de la información y de la comunicación.

\section{Referencias}

Acevedo SuÁrez, Aurymayerly y Zaida Rojas Castillo (2016). «Generalidades del conflicto, los procesos de paz y el posconflicto». Revista de la Facultad de Derecho y Ciencias Políticas, 46 (124): 33-45. DOI: 10.18566/rfdcp.v46n124.ao3.

Álvarez, Alicia, Omar Sandoval y Mildred Puello (2018). «Estrategias pedagógicas para desarrollar competencias ciudadanas en estudiantes de Derecho». Espacios, 39 (29). Disponible en https://bit.ly/3z6GCYr. 
ÁvilA, Ariel y Juan Velasco (2012). «Parapolíticos, narcos, guerrilleros y votos: Revisitando el problema de la teoría democrática a partir del caso colombiano». Papel Político, 17 (2): 371-421. Disponible en https://bit.ly/3eAanaG.

Betancourt, Milthon, Jayson Bernate, Ingrid Fonseca y Luís Rodríguez (2020). «Revisión documental de estrategias pedagógicas utilizadas en el área de la educación física, para fortalecer las competencias ciudadanas». Retos, 83: 845-851. DOI: 10.47197/retos.v38i38.74918.

Capera, José y José Nañes (2017). «Una perspectiva crítica sobre la violencia en Colombia en épocas del postconflicto». Utopía y Praxis Latinoamericana, 22 (78). Disponible en https://bit.ly/3EBXKpS.

Castellanos, Jorge (2020). «Innovación docente y TIC desde la perspectiva de la docencia en Derecho». Revista Pedagogía Universitaria y Didáctica del Derecho, 7(2), 167-184. DOI:10.5354/0719-5885.2020.57150

Cediel Gómez, Yulia, Giohanny Olave Arias, y Mireya Cisneros Estupiñán (2019). «Argumentación para la paz: Avances y desafíos para su enseñanza, como parte de los acuerdos sobre participación política entre el Estado colombiano y las FARCEP». Análisis Político, 32 (95): 23-41. Disponible en 10.15446/anpol.v32n95.80827.

CHAux, Enrique (2009). «Citizenship competencies in the midst of a violent political conflict: the Colombian educational response». Harvard Educational Review, 79 (1): 84-167. Disponible en 10.17763/haer.79.1.d2566q027573h219.

Chaux, Enrique, Ana Velásquez, Juanita Lleras y Patricia Jaramillo (2004). Competencias ciudadanas: De los estándares al aula, una propuesta de integración a las áreas académicas. Bogotá: Universidad de los Andes.

Colonna D'Istria, Pauline (2016). «Del conflicto al agonismo: La operación clave de la democracia». Revista de la Academia, 22: 21-35. DOI: 10.25074/0196318.0.279.

Díaz Perdomo, María y Nelson Rojas Suarez (2018). «Educación para la ciudadanía en el posacuerdo». Eleuthera, 20: 13-34. DOI: 10.17151/eleu.2019.20.2.

Garay Montañez, Nilda (2014). «Investigación y docencia en derecho constitucional: Apuntes y materiales para la comprensión de un constitucionalismo inclusivo». Revista de Educación y Derecho, 11: 1-39. DOI: 10.1344/re\&d.voi11.11989.

Gobierno de Colombia y Fuerza Armada Revolucionaria de Colombia (2016). Acuerdo final para la terminación del conflicto y la construcción de una paz estable y duradera. Disponible en https://bit.ly/3Eyu5Op.

Gómez, Víctor y Valentín Gavidia Catalán (2015). «Describir y dibujar en ciencias. La importancia del dibujo en las representaciones mentales del alumnado». Revista Eureka sobre Enseñanza y Divulgación de las Ciencias, 12 (3): 441-455. Disponible en https://bit.ly/3z63LKm.

HincAPIÉ, Sandra (2017). «Capacidades socioestatales para la democracia local en contextos de violencia en Colombia y México». European Review of Latin American and Caribbean Studies, 103: 71-90. DOI: 10.18352/erlacs.10159. 
LeIva Ramírez, Eric, William Guillermo Jiménez y Orlando Meneses Quintana (2019). «Los derechos fundamentales de la Constitución Política de 1991 como resultado de un proceso constituyente deliberativo». Revista Derecho del Estado, 42: 149-180. DOI: 10.18601/01229893.n42.06.

Luquez De Camacho, Petra, Otilia Fernández, Idania Sansevero y Narbelina Fontanilla (2012). «Praxis de competencias ciudadanas en el ejercicio de la Responsabilidad Social Universitaria». Opción, 28 (69): 480-497. Disponible en https://bit. ly/32tb4QG.

Carrillo Mardones, Olga, Pedro Jurado de los Santos y Patricia Lagos Rebolledo (2018). «Las competencias ciudadanas en la formación profesional de las ciudades de concepción (Chile) y Barcelona (España)». Revista Española de Orientación y Psicopedagogía, 29 (3): 110-130. DOI: 10.5944/reop.vol.29.num.3.2018.23324.

Marina, José y Bernabeu Merlo (2014). Competencia social y ciudadana. Madrid: Alianza.

Maussa, Esnares y Sandra Villareal (2015). «Estrategias pedagógicas aplicadas al desarrollo de competencias ciudadanas en jóvenes universitarios». Opción, 31 (6): 555-576. Disponible en https://bit.ly/3pBfPQT.

Ministerio de Educación Nacional de Colombia (2004). Estándares básicos de competencias en Lenguaje, Matemáticas, Ciencias y Ciudadanas: Guía sobre lo que los estudiantes deben saber y saber hacer con lo que aprenden. Disponible en https://bit.ly/32Im9x1.

PÉREZ, Tito Hernando (2014). «Colombia: De la educación en emergencia hacia una educación para el posconflicto y la paz». Revista Interamericana de Investigación, Educación y Pedagogía, 7 (2). DOI: 10.15332/s1657-107X.2014.0002.06.

Rodríguez Pico, Clara Rocío (2015). «Participación política en los acuerdos de paz de la habana: El prerrequisito de la refrendación de los acuerdos». Análisis Político, 28 (85): 57-75. DOI: 10.15446/anpol.v28n85.56247.

Puerta, Laura y Yaneth Urrego (2016). «Programa en funciones ejecutivas para promover las competencias ciudadanas en educación básica primaria». Revista de Pedagogía, 37 (101): 125-146. Disponible en https://bit.ly/3JszyKa.

Ochman, Marta y Jesús Cantú Escalante (2013). «Sistematización y evaluación de las competencias ciudadanas para sociedades democráticas». Revista Mexicana De Investigación Educativa, 18 (56): 63-89. Disponible en https://bit.ly/32yKnKf.

Toвón Quintero, Gabriel y Gloria Inés Restrepo (2011). «Erradicación de cultivos ilícitos y desplazamiento forzado en el Parque Natural Sierra de La Macarena». Cuadernos de Desarrollo Rural, 6 (63): 31. Disponible en https://bit.ly/3FHZjnF.

Trigos Carrillo, Lina, Laura Fonseca y Natalia Reinoso (2020). «Social impact of a transformative service-learning experience in a post-conflict setting». Front Psychol, 11 (47). DOI: 10.3389/fpsyg.2020.00047. 
URIBE, María Victoria (2019). «El ser ahí de las niñas campesinas durante la violencia en Colombia». Ideas y Valores, 68 (5): 151-162. DOI: 10.15446/ideasyvalores. v68n5Supl.80523.

Zambrano, Elizabeth Leonor (2018). «Prácticas pedagógicas para el desarrollo de competencias ciudadanas». Revista Electrónica de Investigación Educativa, 20 (1): 69-82. DOI: 10.24320/redie.2018.20.1.1409.

\section{Agradecimientos}

Este trabajo fue desarrollado en el grupo Abogados Solidarios UCC de la Universidad Cooperativa de Colombia dentro del proyecto «La percepción del posconflicto en los habitantes del Departamento del Meta (2016-2018) caso: Vista Hermosa». Contó con la participación de los estudiantes del semillero de investigación, los abogados solidarios Leonar Ernesto Lucero Sánchez y Julian Esteban Morales Acosta.

\section{Sobre las autoras}

Yudy Andrea Carrillo Cruz es abogada de la Universidad Libre, candidata a doctora en Derecho de la misma universidad, magíster en Derecho Administrativo, especialista en Derecho Constitucional y Parlamentario, y especialista en docencia universitaria. Además, es profesora tiempo completo, asistente magister en la Universidad Cooperativa de Colombia. También es líder del grupo de investigación Abogados solidarios UCC e investigadora junior reconocida por el Ministerio de Innovación, Ciencia y Tecnología de Colombia. Su correo electrónico es yudy.carrillo@ campusucc.edu.co. (DD https://orcid.org/oooo-0002-1125-1273.

Laura Mercedes Marín Ochoa es abogada de la Universidad del Meta, maestrante en Paz, Desarrollo y Ciudadanía, especialista en contratación estatal. Además, es profesora instructora en la Facultad de Derecho de la Universidad Cooperativa de Colombia. Su correo electrónico es lauram.marin@campusucc.edu.co. (ID https:// orcid.org/oooo-0002-1612-8127. 
La Revista Pedagogía Universitaria y Didáctica del Derecho (RPUDD) es una publicación científica semestral que contribuye a la reflexión multidisciplinaria sobre pedagogía universitaria y didáctica del derecho, para la formación y consolidación de esta área de investigación; así como a la difusión de prácticas innovadoras en la enseñanza-aprendizaje del derecho considerando el contexto nacional e internacional. Es una publicación electrónica internacional con una codirección entre Brasil y Chile.

\author{
DIRECTORA \\ María Francisca Elgueta Rosas \\ Universidad de Chile \\ DIRECTOR \\ Renato Duro Dias \\ Universidad Federal de Rio Grande, Brasil \\ SITIO WEB \\ pedagogiaderecho.uchile.cl \\ CORREO ELECTRÓNICO \\ rpedagogia@derecho.uchile.cl \\ LICENCIA DE ESTE ARTÍCULO
}

Creative Commons Atribución Compartir Igual 4.o Internacional

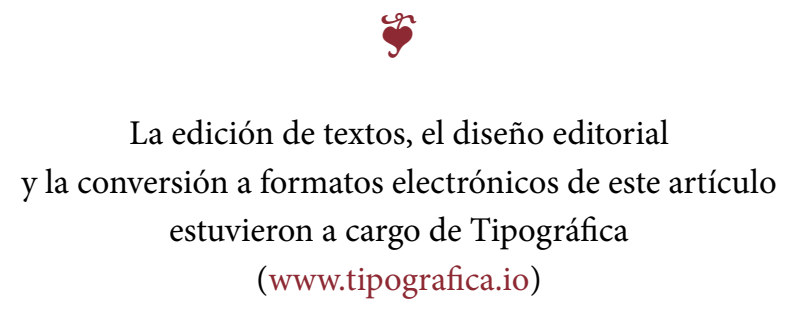

